\title{
Author's reply: Association of D222G substitution in haemagglutinin of 2009 pandemic influenza A (H1N1) with severe disease
}

A Kilander ${ }^{1}$, R Rykkvin ${ }^{1}$, S G Dudman¹, 0 Hungnes (olav.hungnes@fhi.no)1

1. Department of Virology, Norwegian Institute of Public Health, Oslo, Norway

Citation style for this article:

Citation style for this article: Kilander A, Rykkvin R, Dudman SG, Hungnes O. Author's reply: Association of D222G substitution in haemagglutinin of 2009 pandemic influenza A (H1N1) with severe disease. Euro Surveill. 2010;15(14):pii=19535. Available online: http://www.eurosurveillance.org/ViewArticle.aspx?Articleld=19535

This article has been published on 8 April 2010

To the editor: We appreciate the response to our paper made by Dr. Mak and colleagues, whose data from Hong Kong SAR appear to be in good agreement with what we have seen in Norway.

In our original study, we observed a significantly higher frequency of D222G in patients with severe outcomes (including fatal) compared to patients with mild disease. In fact, in both our data set and the Hong Kong data, mutant viruses were not found among several hundred mild cases. Furthermore, as can be seen from our published data, the frequency may be higher also in fatal outcomes (eight of 27 cases) versus severe nonfatal outcomes (three of 34 cases). Comparing these frequencies results in $p=0.078$ with Fisher's exact test (two-sided) and $p=0.046$ with the Mid-P Exact test (two-sided). It would be interesting to know if the new data from Hong Kong SAR can corroborate this observation. Mak et al. report four fatal D222G cases and five non-fatal severe D222G cases, but one would also need to know the total number of fatal cases versus non-fatal severe cases analysed to make the comparison. Hopefully, this information can be obtained.

D222G substitution in virus isolates only and not in the original clinical specimens was found in one case in Hong Kong and 14 cases reported by the United States (US) Centers for Disease Control and Prevention [1]. We have also seen this virus culture artefact in one case with mild disease. This case was counted as wild type in our data set. This further underscores the importance to perform the sequence analysis of the primary specimen.

The frequency of $\mathrm{D} 222 \mathrm{G}$ mutant viruses in the severe cases is somewhat lower in the Hong Kong data, compared to ours (4.1 per cent in Hong Kong data versus 18 per cent in our data set). Whilst this difference may represent a real variation in frequency, it may also arise from a different composition of cases, e.g. if the proportion of fatal cases were higher in the Norwegian sample of severe plus fatal cases. Mak and colleagues also observed, as we did, that the $222 \mathrm{G}$ mutant sometimes occurs in a mixture with non-mutated $222 \mathrm{D}$ genomes. Sensitivity of detection of mutant viral genomes when occurring as the minority variant in such mixtures may thus also influence the observed frequency. We have been using a pyrosequencing assay to identify the 222 genotype. Under ideal conditions this methodology can reliably detect and quantitate a mutant when present in the total virus population at levels as low as $10 \%$ [2]. However, in our data set the initial finding of D222G mutants by pyrosequencing could uniformly be verified by conventional sequencing. Therefore, since the Hong Kong data come from a study focusing on this particular position we assume that the methodology difference is not likely to have caused the different frequencies in the two data sets. In the overall global data, however, it is possible that some cases with mutant/wild type mixtures have been overlooked and only the majority sequence recorded.

References

1. World Health Organization. Preliminary review of D222G amino acid substitution in the haemagglutinin of pandemic influenza $\mathrm{A}\left(\mathrm{H}_{1} \mathrm{~N}_{1}\right) 2009$ viruses. Wkly Epidemiol Rec. 2010;85(4):21-2. [English, French].

2. Lackenby A, Democratis J, Siqueira MM, Zambon MC. Rapid quantitation of neuraminidase inhibitor drug resistance in influenza virus quasispecies. Antivir Ther. 2008;13(6):809-20. 